\title{
A Giant Vertebral Aneurysm Presented with Cough and Dyspnea and Mistreated Over a Year: An Extraordinary Case Report and Review of the Literature
} \author{
DAGLIOGLU, MD \\ ${ }^{1}$ Department of Neurosurgery, Ankara Bilkent City Hospital, Ankara, Turkey \\ ${ }^{2}$ Department of Interventional Radiology, Ankara Bilkent City Hospital, Ankara, Turkey
}

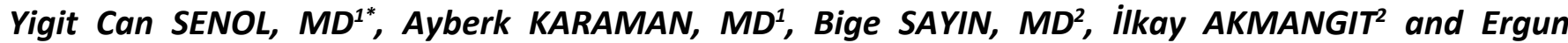

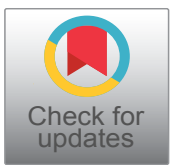

*Corresponding author: Yigit Can SENOL, Department of Neurosurgery, Ankara City Hospital, Neurology and Orthopedics MH3 $4^{\text {th }}$ Floor, 06800, Cankaya/Ankara/Turkey, Tel: +90-539-702-4602; +90-312-552-6000

\begin{abstract}
We report an exceptional case of vertebral artery aneurysm presented with dyspnea and cough symptoms which is misdiagnosed with bronchiectasis. Literature showed that cough can be an exceptional symptom of vertebral aneurysms.

Vertebral aneurysms presented with cough are rare and might be confused with other lung diseases such as pneumonia, chronic obstructive lung disease (COPD) or bronchiectasis.

Introduction: Giant intracranial aneurysms are rare entities defined by a diameter of at least $25 \mathrm{~mm}$, accounting for $5 \%$ of all cases of intracranial aneurysm. These aneurysms can treat with endovascular; flow diverters, stents, primary coiling and combined treatments and vascular procedures such as clipping, or bypass surgeries. In the present case report, because of the main symptoms of cough and dyspnea, aneurysm diagnoses are delayed and the patient had a treatment of bronchiectasis over a year.
\end{abstract}

Conclusion: Cough and dyspnea can be the only symptoms of the aneurysms. Vertebral aneurysm should be in the differential diagnoses of coughs that doesn't alleviate.

\section{Keywords}

Vertebral artery aneurysm, Endovascular treatment, Cough, Dyspnea

\section{Introduction}

Giant intracranial aneurysms are rare entities defined by a diameter of at least $25 \mathrm{~mm}$, accounting for $5 \%$ of all cases of intracranial aneurysm [1]. As their nature of rarity giant aneurysms are less seen in posterior circulation and associated with high mortality and morbidity [2]. Due to small size of posterior fossa, posterior circulation aneurysms can present with cranial nerve palsies, hydrocephalus, motor impairment therefore substantial mass effect. Because of the mass effect, infrequently vertebral artery aneurysm can confer with cough and dyspnea due to vagal nerve deterioration [3]. Although, anterior circulation aneurysm can treat with endovascular; flow diverters, stents, primary coiling and combined treatments and vascular procedures such as clipping, or bypass surgeries also posterior circulation can be treated with same methods on the other hand they have poor outcomes when we compare with the anterior circulation aneurysms. Regarding the giant and complexity of aneurysms cannot be managed with surgical strategies, Flow diversion such as pipeline embolization devices with coil assistance is an alternative treatment [4]. However, the mortality and morbidity rates of posterior circulation aneurysm treated using flow diversion devices are much higher compared with those of pre-circulation aneurysm [5]. In the present case report, because of the main symptoms of cough and dyspnea aneurysm diagnosis is delayed and the patient had a treatment of bronchiectasis over a year.

Citation: SENOL YC, KARAMAN A, SAYIN B, AKMANGIT I, DAGLIOGLU E (2021) A Giant Vertebral Aneurysm Presented with Cough and Dyspnea and Mistreated Over a Year: An Extraordinary Case Report and Review of the Literature. Neurosurg Cases Rev 4:091. doi.org/10.23937/26434474/1710091

Accepted: November 27, 2021; Published: November 29, 2021

Copyright: (c) 2021 SENOL YC, et al. This is an open-access article distributed under the terms of the Creative Commons Attribution License, which permits unrestricted use, distribution, and reproduction in any medium, provided the original author and source are credited. 

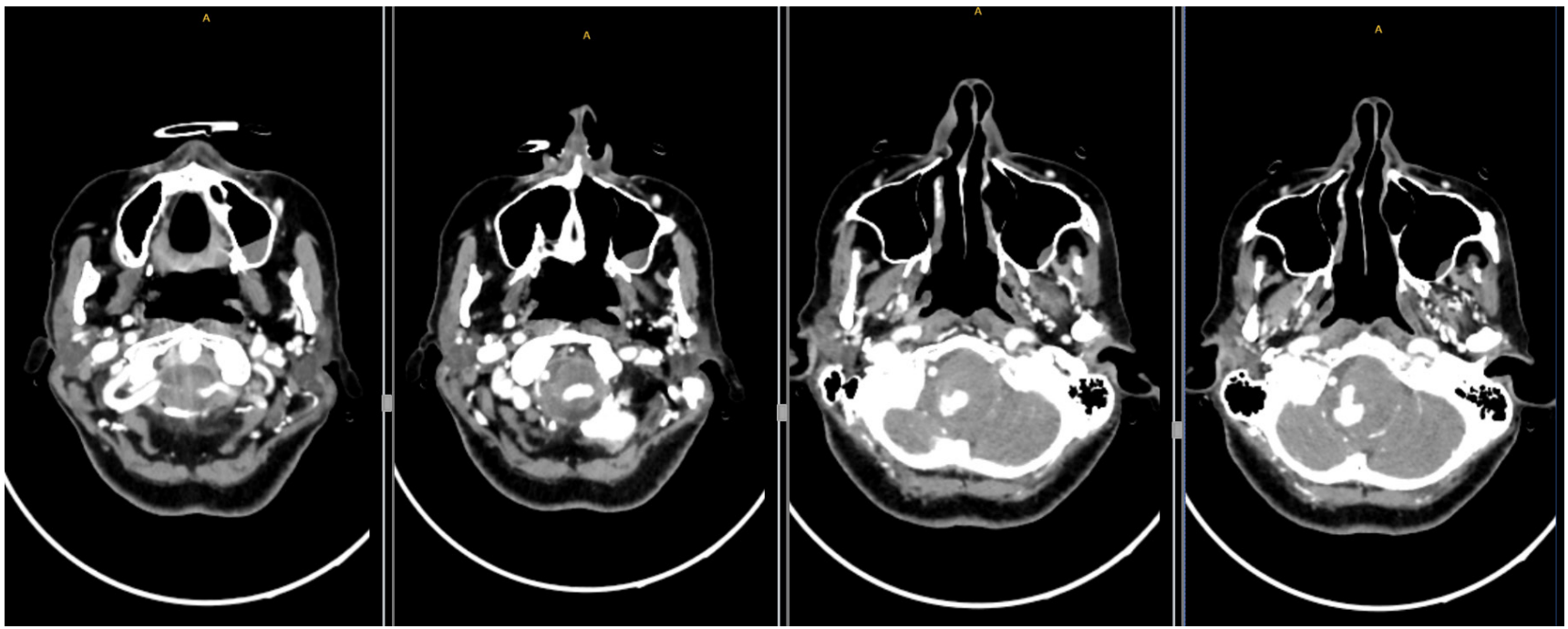

Figure 1: Ct scans revealed a large vertebrobasilar aneurysm and obvious compression of the brainstem.
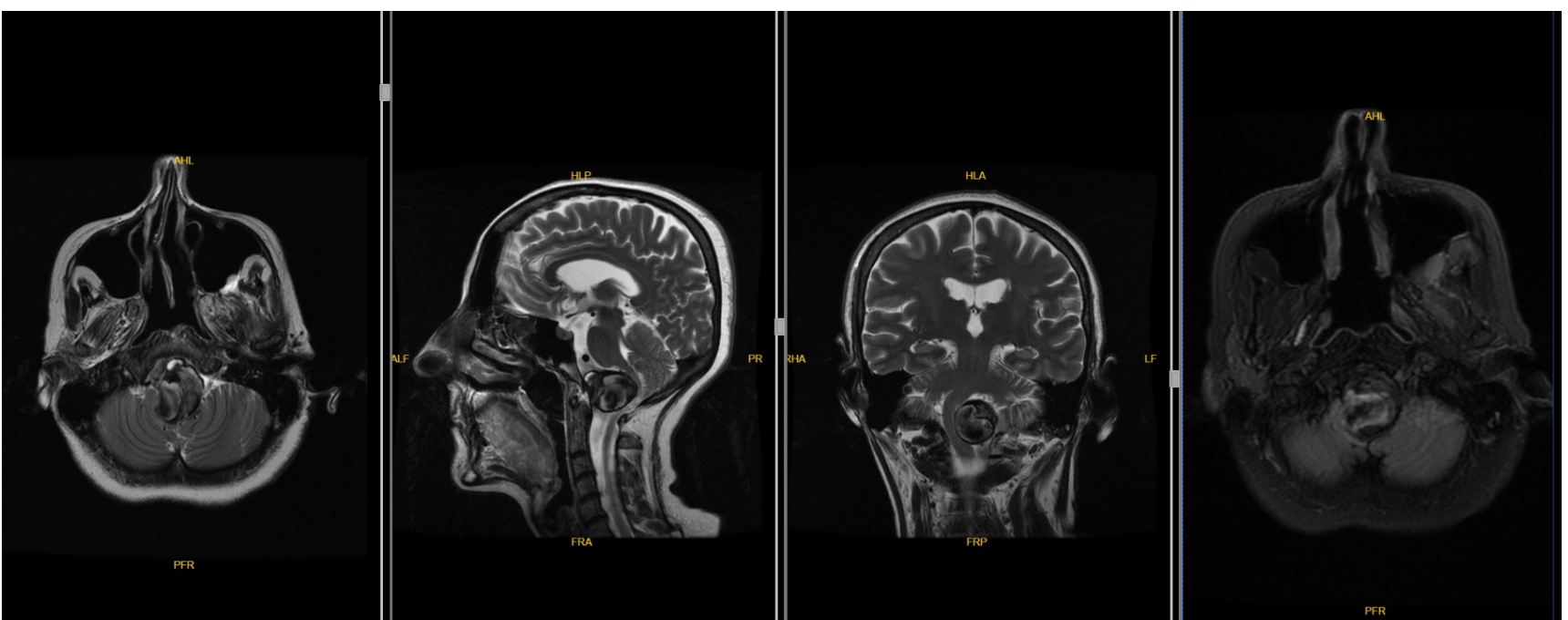

Figure 2: A giant spherical aneurysm right vertebral V4 segment aneurysm with a maximum diameter of $30 \mathrm{~mm}$ was identified.

\section{History and Examination}

The patient was a 47-year-old female admitted to Ankara Bilkent City Hospital (Ankara, Turkey) in June 2018, who had been experiencing symptoms of cough, dyspnea and hoarseness for 1 year. Her cough was treated with several antibiotics and diagnosed with bronchiectasis but there was no improvement of her complaints. In June 2019 because of the progression of her dyspnea, admitted to our hospital's emergency service. Her tachypnea got worse and saturation fell down. At last, the patient has elective intubation in the E.R. On the elective intubation, E.R doctor noticed that the patient's left vocal cord was paralyzed and an emergent head and cervical computer tomography angiography performed. Ct scans revealed a large vertebrobasilar aneurysm and obvious compression of the brainstem (Figure 1). Patient transferred to a neurosurgery intensive care unit and started antibiotic of teicoplanin with the loading dose of $400 \mathrm{mg} 2 \times 1$ and continued $400 \mathrm{mg} 1 \times 1$.
Physical examination was performed before intubation by the E.R. doctor and showed an obvious right-slant tongue extension, right sided walking with Medical Research Council [6] grade 5- muscle strength in the right-limb and negative bilateral finger-to-nose and heel-to-knee tibia tests with normoactive reflexes. After admission to the ICU, the patient underwent digital subtraction angiography (DSA) at Ankara Bilkent City Hospital. A giant spherical aneurysm right vertebral V4 segment aneurysm with a maximum diameter of 30 $\mathrm{mm}$ was identified (Figure 2).

\section{Treatment Strategy}

Informed consent was provided by the patient and her relatives prior to endovascular therapy. A treatment plan was formulated after angiography. First, a microcatheter (headway 17) was placed through the right vertebral artery and the Pipeline was completely released through the right VA. Subsequently, the aneurysm was relatively tightly embolized with coils. Clopidogrel was administered with a loading dose of 300 
mg and continued with $75 \mathrm{mg}$ per day. The Clopidogrel resistance test was evaluated with $72 \%$ on day 4 . At 5 days following hospitalization, the giant aneurysm of the V4 segment was embolized with Pipeline $5 \times 35$ $\mathrm{mm}$ flow diverter device. Stagnation of blood in the aneurysm was seen in control angiograms.

For 2 days following the endovascular treatment, the patient was listless and complained of a headache worse than those that had occurred during the pre-embolization period. Physical examination revealed no new positive signs. Postoperative the patient transferred to the neuro-ICU and on post-operative day 2 her vitals were improved and extubated. Antibiotics were stopped on the day 10 of the admission. The patient was transferred to the Neurosurgery inpatient service at the day of 12 of her admission.

\section{Follow-up}

After 2 months of the operation, the patient had increased white phlegm and her choking cough was continuing. Furthermore, the patient's right limb muscle strength was improved compared with that pre-embolization and the patient has Medical Research Council grade 5 muscle strength in the right-limb. Three months after embolization, re-examination of the head MRI and MRA revealed that the aneurysm was completely occluded [Raymond-Roy classification, Class I [7]]; although, the brainstem was still compressed when comparing with the pre-embolization Mri (Figure 3). At 12 months after surgery, her phlegm was markedly improved and discontinued with the medical drugs. Cough and hoarseness disappeared. Re-examination of DSA revealed no aneurysm recurrence [Raymond-Roy classification, Class I [7]] and no stenosis in the parent artery on MRI (Figure 4) and computer tomography angiography (Figure 5). The modified Rankin scale score [8] of the patient is 1 point.

\section{Discussion}

Flow diverters are a more widely accepted tool for aneurysm treatment within the last decade.

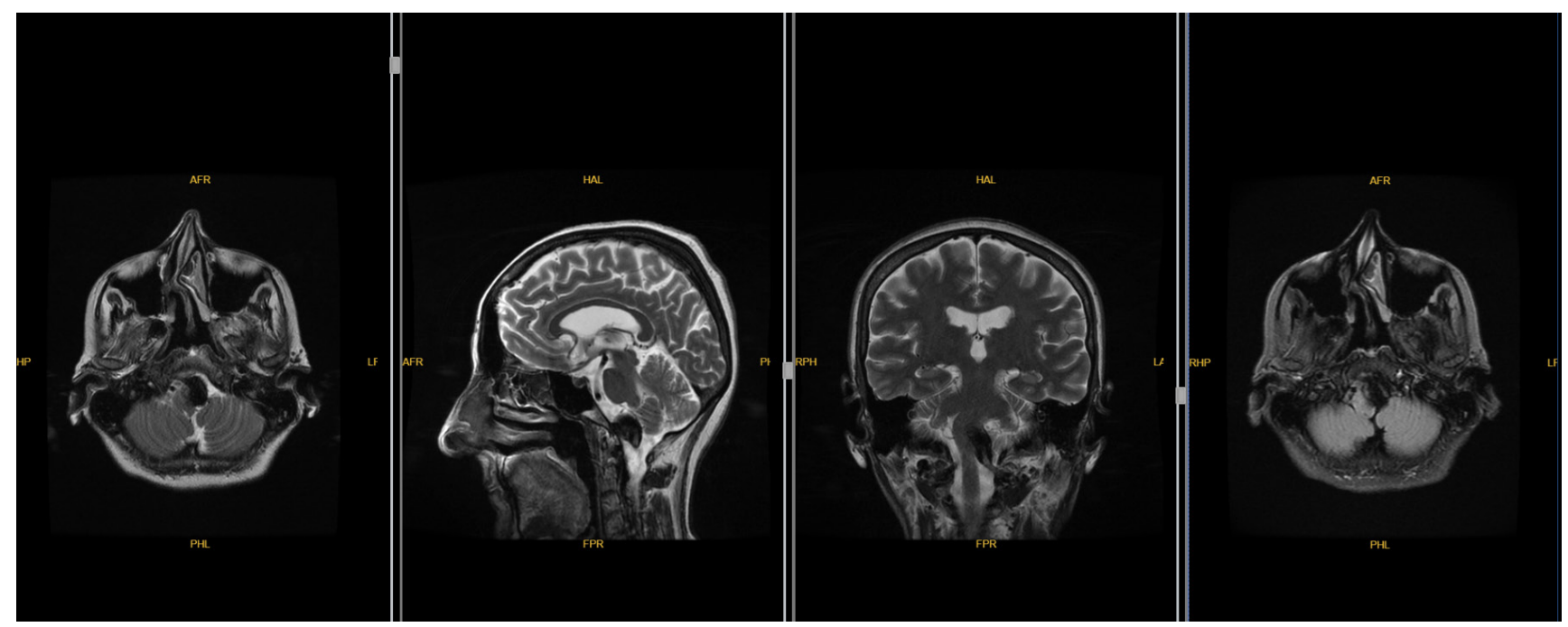

Figure 3: At 12 months after surgery, her phlegm was markedly improved and discontinued with the medical drugs.
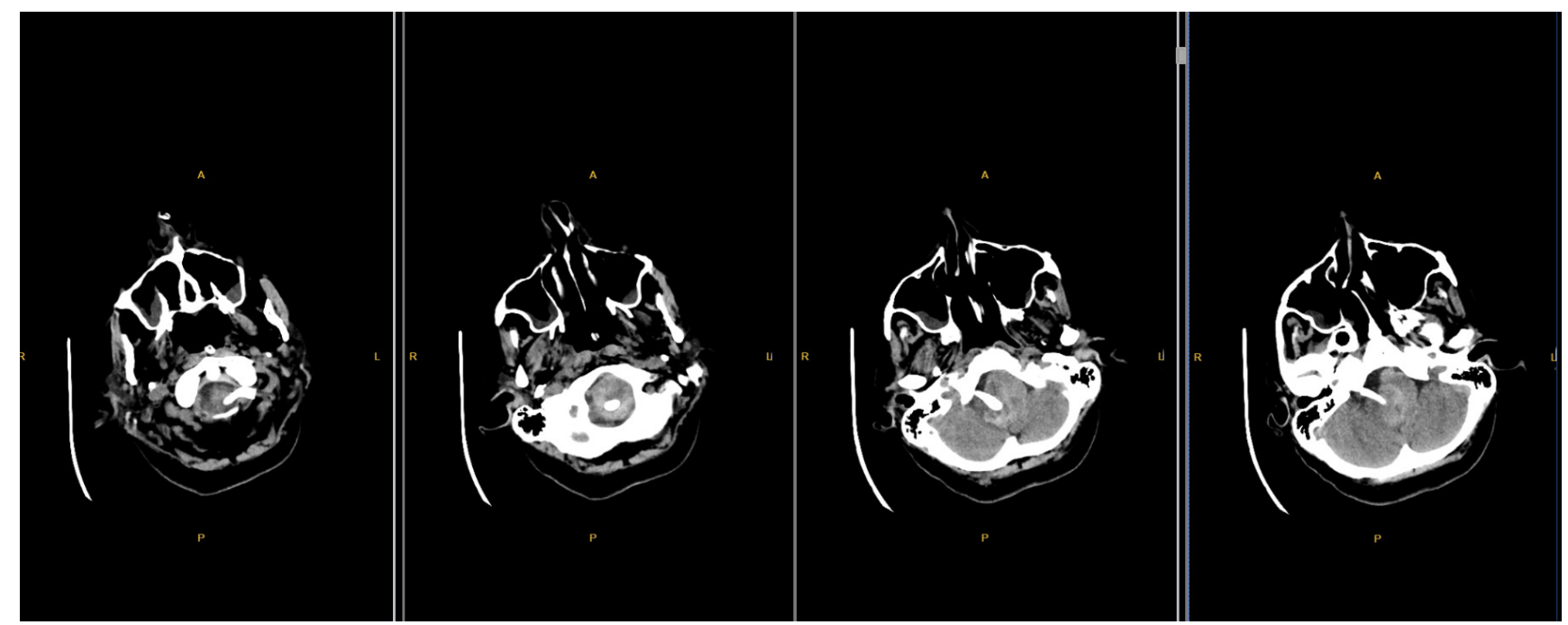

Figure 4: No stenosis in the parent artery on MRI. 

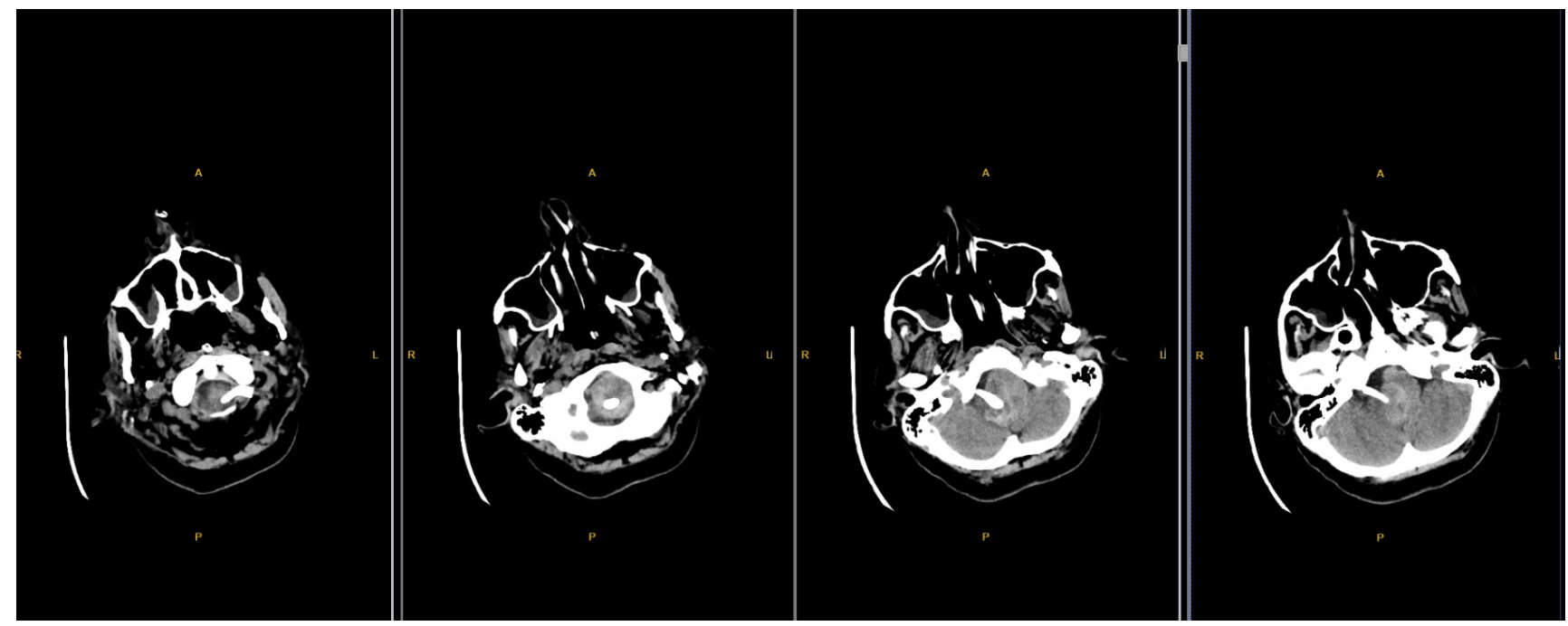

Figure 5: Computer tomography angiography.

A recent meta-analysis by $\mathrm{Ye}$, et al. [5] reported an aneurysm occlusion rate of $78.8 \%$ after an average follow-up of 6.3 months, and neurological morbidity and mortality rates after FDD treatment were 9.8 and $3.8 \%$, respectively. The rupture rate of giant, unruptured, posterior circulation aneurysms within 5 years may be up to $50 \%$ [8]. The relevant factors to be considered when treating such complex aneurysms include the shape, location, vascular architecture and collateral circulation of the aneurysm's parent artery. Treatment of these aneurysms has always posed a great challenge for neurosurgery. Though, the complication rate (the number of complications divided by the number of patients) of complex posterior circulation aneurysms treated with flow diversion devices is significantly higher compared with that of anterior circulation aneurysms, reconstruction of the parent artery with coil embolization is a suitable alternative when surgical treatment fails. Up To date, only a small number of studies have reported on the treatment of giant aneurysm at the VBJ. Liang, et al. [8] described the endovascular treatment of 99 patients with large or giant aneurysms of the posterior circulation, 12 of which were located at the VBJ; 3 of those 12 cases were treated by occluding the contralateral VA with coils, and the bilateral VA was sacrificed in 1 of the 12 cases. Beyond shadow of doubt, cough and dyspnea can be the only symptoms of the aneurysms. Vertebral aneurysm should be in the differential diagnoses of coughs that doesn't alleviate.

\section{Statements}

This report followed guidelines to be HIPAA compliant and permission was obtained from the patient to publish identifiable photographs. Written informed consent was obtained from the patient for publication of this case report and accompanying images. The study adhered to the tenets of the Declaration of Helsinki.

\section{Conflict of Interest Statement}

None of the authors have a proprietary interest in this study or any conflicts of interest to disclose.

\section{Funding Sources}

We declare no financial support of this case report.

\section{Author Contributions}

The authors confirm contribution to the paper as follows: study conception and design: Ergun Daglioglu and Ilkay Akmangit; data collection: Yigit Can Senol and Ayberk Karaman. Draft manuscript preparation: Bige Sayın. All authors reviewed the results and approved the final version of the manuscript.

\section{References}

1. Haemmerli J, Lenga $P$, Hong $B$, Kursumovic A, Maldaner $\mathrm{N}$, et al. (2019) Clinical implications and radio- graphic characteristics of the relation between giant intracranial aneurysms of the posterior circulation and the brainstem. Acta Neurochir (Wien) 161: 1747-1753.

2. Awad AJ, Mascitelli JR, Haroun RR, De Leacy RA, Fifi JT, et al. (2017) Endovascular management of fusiform aneurysms in the posterior circulation: The era of flow diversion. Neurosurg Focus 42: E14.

3. Rizzo L, Crasto SG, Savio D, Veglia S, Davini O, et al. (2006) Dissection of cervico-cephalic arteries: Early diagnosis and follow-up with magnetic resonance imaging. Emerg Radiol 12: 254-265

4. Aguilar Perez M, Henkes E, Hellstern V, Serna Candel C, Wendl C, et al. (2021) Endovascular treatment of anterior circulation aneurysms with the p64 flow modulation device: Mid- and long-term results in 617 aneurysms from a single center. Oper Neurosurg (Hagerstown).

5. Ye G, Zhang M, Deng L, Chen X, Wang Y (2016) Meta-analysis of the efficiency and prognosis of intracranial aneurysm treated with flow diverter devices. J Mol Neurosci 59: 158-167.

6. Raymond J, Guilbert F, Weill A, Georganos SA, Juravsky L, et al. (2003) Long-term angiographic recurrences after selective endovascular treatment of aneurysms with detachable coils. Stroke 34: 1398-1403. 
7. Van Swieten JC, Koudstaal PJ, Visser MC, Schouten HJ, van Gijn J (1988) Interobserver agreement for the assessment of handicap in stroke patients. Stroke 19: 604-607.
8. Liang $\mathrm{F}$, Zhang $\mathrm{Y}$, Yan $\mathrm{P}, \mathrm{Ma} \mathrm{C}$, Liang $\mathrm{S}$, et al. (2019) Predictors of periprocedural complications and angiographic outcomes of endovascular therapy for large and giant intracranial posterior circulation aneurysms. World Neurosurg 125: e378-e384. 